\title{
Genetic diversity of caprine Blastocystis from Peninsular Malaysia
}

\begin{abstract}
Blastocystis sp. is a common intestinal parasite found in humans and animals. The possibility of zoonotic transmission to humans from livestock especially goats led us to investigate the genetic diversity of caprine Blastocystis sp. obtained from five different farms in Peninsular Malaysia. Moreover, there is a lack of information on the prevalence as well as genetic diversity of Blastocystis sp. in goat worldwide. Results showed that 73/236 (30.9\%) of the goats were found to be positive for Blastocystis infection. The most predominant Blastocystis sp. subtype was ST1 (60.3\%) followed by ST7 (41.1\%), ST6 (41.1\%), and ST3 (11.0 \%) when amplified by PCR using sequenced-tagged site (STS) primers. Four farms had goats infected only with ST1 whereas the fifth showed mixed infections with multiple STs. The proximity of the fifth farm to human dwellings, nearby domesticated animals and grass land as opposed to a sterile captive environment in the first four farms may account for the multiple STs seen in the fifth farm. Since ST1, ST3, ST6 and ST 7 were previously reported in human infection worldwide in particular Malaysia, the potential of the zoonotic transmission of blastocystosis should not be disregarded. The implications of different farm management systems on the distribution of Blastocystis sp. STs are discussed.
\end{abstract}

Keyword: Blastocystis; Goats; Malaysia 